\title{
Utilidad percibida de la educación para la salud en adolescentes con cateterismo cardiaco y sus cuidadores primarios ${ }^{1}$
}

\section{Perceived usefulness of health education for adolescents undergoing cardiac catheterization and their informal primary caregivers}

\author{
Julieta Beatriz Candelario-Mosco², Rodrigo Hiroshi González-Luna³, \\ Alejandro Flores-Arizmendi ${ }^{4}$ y Angélica Riveros-Rosas ${ }^{5}$
}

\begin{abstract}
Citación: Candelario-Mosco J.B., González-Luna, R.H., Flores-Arizmendi, A. y Riveros-Rosas, A. (2020). Utilidad percibida de la educación para la salud en adolescentes con cateterismo cardiaco y sus cuidadores primarios. Psicología y Salud, 30(2), 189-198. https://doi.org/10.25009/pys. v30i2.2653.
\end{abstract}

\section{RESUMEN}

\begin{abstract}
Se ha utilizado eficazmente la educación para la salud para mejorar el autocuidado en las intervenciones médicas en virtud de que informa a los pacientes sobre acciones específicas que facilitan su recuperación. Objetivo: Analizar la utilidad percibida de la educación para la salud en adolescentes con cateterismo cardiaco y sus cuidadores primarios. Método: Participaron 32 adolescentes y sus cuidadores primarios en dos grupos: uno con atención habitual y otro con educación para la salud. Resultados: $69 \%$ de los adolescentes y $85 \%$ de los cuidadores primarios del grupo de atención habitual y $100 \%$ de los adolescentes y cuidadores primarios del grupo con educación para la salud consideraron que recibir esta última antes del cateterismo cardiaco les fue de gran utilidad para generar un mejor conocimiento acerca de la etapa previa, el transcurso y la etapa posterior al cateterismo y los cuidados necesarios, así como para lograr una mejor autorregulación emocional, todos ellos elementos que les proporcionaron una sensación de mayor control y la posibilidad de acción durante su recuperación. Discusión: Si bien la educación para la salud se consideró útil y con un efecto positivo, se discute la importancia de respetar el deseo de los pacientes y sus familias acerca de recibirla o no.
\end{abstract}

Palabras clave: Educación para la salud; Adolescentes; Cuidadores primarios; Autocuidado.

\begin{abstract}
Health education has been successfully used to improve self-care during hospital medical interventions, since it provides participants with specific actions to support their recovery. The objective of the present study was to explore the perceived effects and value of health education on adolescents who received a cardiac catheterism and on their primary informal caregivers. Method. A total of 32 adolescents and their primary informal caregivers participated and were assigned to one of
\end{abstract}

\footnotetext{
${ }^{1}$ El presente estudio se deriva parcialmente de la tesis doctoral del primer autor bajo la dirección del último. Los autores agradecen a los pacientes, cuidadores primarios y personal médico y de enfermería del Centro Médico Nacional 20 de Noviembre, así como al apoyo del Consejo Nacional de Ciencia y Tecnología (CONACYT), con número de becario 583957 y CVU 698479, que hicieron posible este estudio. Artículo recibido el 11 de junio y aceptado el 24 de octubre de 2019.

${ }^{2}$ Centro Médico Nacional 20 de Noviembre del Instituto de Seguridad y Servicios Sociales de los Trabajadores del Estado, Félix Cuevas 540, Col del Valle Sur, 03100 Ciudad de México, México, y Facultad de Psicología, Universidad Nacional Autónoma de México, correo electrónico: julieta_beatrizcm@hotmail.com.

${ }^{3}$ Hospital Regional General Ignacio Zaragoza del Instituto de Seguridad y Servicios Sociales de los Trabajadores del Estado, Calzada Ignacio Zaragoza 1711, Col. Ejército Constitucionalista, Chinam Pac de Juárez, 09220 Ciudad de México, México, y Facultad de Medicina, Universidad Nacional Autónoma de México.

${ }^{4}$ Centro Médico Nacional "20 de Noviembre" del Instituto de Seguridad y Servicios Sociales de los Trabajadores del Estado.

${ }^{5}$ Facultad de Contaduría y Administración, Universidad Nacional Autónoma de México, Av. Universidad 3000, 04510 Ciudad de México, México.
} 
two conditions: one received a specifically designed health education intervention conducted by psychologists and the other half received the usual hospital protocol. Results revealed that $69 \%$ of adolescents and 85\% of primary informal caregivers in the usual hospital protocol stated that the health education intervention would have been useful for them in terms of acquiring knowledge on medical procedures and improving emotional control. In the group that received the health education intervention, $100 \%$ of both catheterized patients and caregivers expressed it was useful because it provided a sense of greater control as well as concrete strategies to support medical recovery such as adhering to indications by the health team. Discussion. Even in the context of an outstanding perception of the value of psychoeducation, health professionals should keep in mind the need to respect the desire of patents and their families regarding the option to receive it or not.

Keywords: Health education; Adolescents; Primary informal caregivers; Self-care.

$\mathrm{E}$ 1 cateterismo cardiaco es un procedimiento diagnóstico y terapéutico que ofrece importantes ventajas médicas al paciente con cardiopatía congénita; sin embargo, los pacientes reportan cardiaco dolor de espalda, malestar urinario y dificultad para mantener el reposo e inmovilidad prolongada en cama, que puede requerir hasta 24 horas, durante el poscateterismo (Rezaei-Adaryani, Ahmadi, Mohamadi y Asghari-Jafarabadi, 2009; Woods, Froelicher, Motzer y Bridges, 2010). En cuanto al aspecto emocional, se observa ansiedad e ira debido a las necesidades insatisfechas al estar en dicha condición (Keeling, Fisher, Haugh, Powers y Turner, 2000; Peterson et al., 2010), lo que genera dificultades para la adherencia terapéutica y la colaboración con el personal de salud. La conducta y actitud del paciente en el poscateterismo cardiaco son muy significativos ya que durante este periodo es el más responsable de los resultados del tratamiento.

En la fase previa y posterior al cateterismo se requiere, por consiguiente, el acompañamiento de un cuidador, que generalmente son los padres, quienes pueden sentirse culpables de la necesidad de dicha intervención en su hijo y responsables de exponerlo al estrés que implica tal procedimiento; con mayor frecuencia las madres experimentan es- trés y sentimientos de impotencia por las posibles complicaciones o resultados en el transcurso y la etapa posterior de dicha intervención (Aranha, Sams y Saldanha, 2016; Kobayashi, Turner, Forbes y Aggarwal, 2018; Mu, Ma, Hwang y Chao, 2002; Utens et al., 2000).

Lo anterior implica que ambos padres tengan dificultad para asumir activamente el papel de ayudar a su hijo a manejar la experiencia, por lo que en algunos casos optan por mantenerse al margen de la situación para no interferir con el quehacer médico, o bien, por el contrario, le brindan el apoyo emocional necesario (Ellerton y Merriam, 1994).

Otro factor que agrava dicha dificultad en los padres es la falta de conocimientos para el mantenimiento de la salud y la prevención de complicaciones. En el estudio de Elsobky, Mohamed y Mohamend (2018), la mayoría de las madres desconocían las señales de alarma que se deben atender en el hospital, como un sonido anormal del corazón; taquicardia; mala alimentación; tumefacción de ojos, manos y pies; incisión dolorosa, enrojecida, hinchada o con fugas de líquido, entre otras.

Se han efectuado diversos estudios destinados a averiguar la precisión de la información de que disponen los pacientes respecto al cateterismo cardiaco, encontrándose en general que la mayoría de ellos carecen de información adecuada sobre el procedimiento (Buffum et al., 2006; Jamshidi, Abbaszadeh y Kaliani, 2010) y, por ende, de comprensión o de percepciones correctas acerca de la intervención y de sus posibles consecuencias, lo que favorece que muestren un considerable aumento de su ansiedad al momento o justo antes del cateterismo (Mitchell, 2003; Samira, Nahid y Samah, 2016). Un factor que puede influir en este fenómeno es que por lo regular el equipo de salud brinda información sobre el estado de salud y el tratamiento principalmente a los padres, no así al paciente adolescente (Lee y Kim, 2010). Cabe destacar que son muy escasos tales estudios en el caso de México y América Latina.

La eficacia de intervenciones tales como la educación para la salud, en la que se brinda información sobre los procedimientos médicos al paciente, se ha evaluado en niños, adolescentes 
(Chair, Chau, Sit, Wong y Chan, 2012; He et al., 2014; Vincent et al., 2012) y padres (Aranha et al., 2016), probándose su evidente efectividad en la reducción de la ansiedad (Abbas y Halima, 2017; Fernandes, Arriaga y Esteves, 2014), en la atención de las necesidades del paciente durante la recuperación en el hogar (como la movilidad, el ejercicio, la relajación, la dieta apropiada y el control adecuado del dolor) (Elsobky et al., 2018) y en la promoción del autocuidado (Rodríguez, Arredondo y Herrera, 2012). Sin embargo, no se ha explorado al parecer el impacto de la educación para la salud en el cateterismo cardiaco en población adolescente latinoamericana en general, ni mexicana en particular.

En virtud de lo angustiante e incómodo del procedimiento, la afectación al autocuidado y la escasa información existente sobre la necesidad y el efecto de la información, el presente estudio tuvo como objetivo conocer la utilidad percibida de la educación para la salud en los adolescentes sometidos a cateterismo cardiaco y en sus cuidadores primarios.

\section{MÉTODO}

Se trató de un estudio exploratorio en adolescentes sometidos a dicha intervención y sus cuidadores primarios, los cuales fueron distribuidos en dos grupos respecto al procedimiento médico: de atención habitual y de educación para la salud, el cual se llevó a cabo en el servicio de Cardiología Pediátrica del Centro Médico Nacional "20 de Noviembre" del Instituto de Seguridad y Servicios Sociales de los Trabajadores del Estado (ISSSTE) de la Ciudad de México, donde los adolescentes estaban programados para practicarles un cateterismo cardiaco a lo largo de un año.

\section{Participantes}

Grupo de educación para la salud (GES): Participaron 19 adolescentes, 11 de ellos de sexo femenino (57.89\%), de entre 11 y 18 años de edad, con una media de 14 y una desviación típica de 2.87 años. Participaron en este mismo grupo 19 cuidadores primarios: 16 madres $(84.21 \%)(\mathrm{M}=48$ años $\mathrm{y}$
D.E. $=5.91)$, quienes, a la par de sus hijos, fueron instruidos a través de la educación para la salud.

Grupo con atención habitual (GAH): Consistió de 13 adolescentes, 7 de los cuales fueron de sexo masculino (54\%), de entre 11 y 18 años de edad, con una media de 14 años de edad y desviación típica de 2.15. Participaron también 13 cuidadores, 8 de los cuales fueron las madres $(61.5 \%)$, con una media de 48 años de edad y desviación típica de 5.93 .

Como criterios de inclusión para los adolescentes se consideró tener entre 11 y 18 años de edad, programados y sometidos a cateterismo cardiaco. Los criterios para los cuidadores primarios fueron que estuvieran al cuidado del paciente la mayor parte del internamiento (como mínimo 70\% del tiempo) y, en el caso de pertenecer al grupo de educación para la salud, que fuera quien hubiese recibido la instrucción el que cuidara del paciente.

Los criterios de exclusión para los adolescentes fueron que el cateterismo cardiaco se reprogramara para otra fecha o no se realizara, así como que tuviera un proceso de recuperación fuera de lo esperado debido a complicaciones. Para los cuidadores primarios, que permanecieran menos de $70 \%$ del tiempo al cuidado del paciente, y que quien hubiera recibido la instrucción para la salud no fuera quien quedase al cuidado del paciente.

\section{Materiales}

Se elaboró un cuestionario con preguntas abiertas a fin de explorar la utilidad de la educación para la salud recibida por los adolescentes y por sus cuidadores primarios. La Tabla 1 contiene un ejemplo de las preguntas hechas a los mismos de acuerdo al tema explorado.

Las preguntas se pilotearon y fueron validadas por expertos en las áreas psicológica y médica, y así también se aseguró la claridad y pertinencia de dichas preguntas con un grupo de adolescentes y sus cuidadores primarios. El grupo de expertos en psicología sugirió el uso de una escala análoga para cuantificar la utilidad de la educación para la salud (donde 0 es el valor inferior y 10 el valor máximo). Se decidió agregar una pregunta sobre la información que les hubiera gustado obtener al identificar que las respuestas de los adolescentes 
y sus cuidadores primarios eran principalmente de orden emocional (como "Que todo va a estar bien, que le echen ganas y que no tengan miedo").
El área médica consideró correctas las preguntas elaboradas.

Tabla 1. Ejemplo de las preguntas hechas a los adolescentes y cuidadores primarios.

\begin{tabular}{|c|c|c|}
\hline Tema explorado & Adolescentes & Cuidadores primarios \\
\hline $\begin{array}{l}\text { Utilidad de la educación para la salud } \\
\text { para quienes la recibieron y como } \\
\text { posibilidad de utilidad para quienes } \\
\text { recibieron atención habitual }\end{array}$ & $\begin{array}{l}\text { Si hubieras tendo la oportunidad de re- } \\
\text { cibir antes de tu procedimiento medico } \\
\text { información al respecto de tu cateteris- } \\
\text { mo cardiaco y de los cuidados que debes } \\
\text { de tener durante tu recuperación en el } \\
\text { hospital ¿crees que te hubiera sido útil?, } \\
\text { ¿qué tanto del } 0 \text { al } 10 \text { ? y ¿en qué forma } \\
\text { te ayudaría no te ayudaría? }\end{array}$ & $\begin{array}{l}\text { Antes del cateterismo cardiaco, usted tuvo } \\
\text { la oportunidad de recibir información } \\
\text { acerca del procedimiento médico (catete- } \\
\text { rismo cardiaco) y de los cuidados que se } \\
\text { deben de tener durante la recuperación de } \\
\text { su hijo en el hospital, ¿cree que ésta fue de } \\
\text { utilidad?, ¿qué tanto del } 0 \text { al } 10 ? \text { ? ¿en qué } \\
\text { forma le ayudó o no le ayudó? }\end{array}$ \\
\hline $\begin{array}{l}\text { Información que le gustaría transmitir } \\
\text { a otros en las mismas condiciones. }\end{array}$ & $\begin{array}{l}\text { Si conocieras a otro adolescente al que } \\
\text { también le van a hacer un cateterismo } \\
\text { cardiaco, ¿qué le dirías, platicarías o } \\
\text { recomendarias? }\end{array}$ & $\begin{array}{l}\text { Si conociera a otro padre de familia que } \\
\text { tiene un hijo adolescente que va a someter- } \\
\text { se a un cateterismo cardiaco ¿qué le diría, } \\
\text { platicaría o recomendaría? }\end{array}$ \\
\hline $\begin{array}{l}\text { Interés en recibir información } \\
\text { de alguien con experiencia directa. }\end{array}$ & $\begin{array}{l}\text { Si antes de tu cateterismo cardiaco hu- } \\
\text { bieras tenido la oportunidad de platicar } \\
\text { con un adolescente que ya vivió un cate- } \\
\text { terismo cardiaco ¿qué le preguntarías? } \\
\text { o ¿qué te gustaría saber? }\end{array}$ & $\begin{array}{l}\text { Si antes del cateterismo crdiaco de su hijo } \\
\text { hubiera tenido la oportunidad de platicar } \\
\text { con un padres de familia que ya vivió un } \\
\text { cateterismo cardiaco de su hijo ¿qué le } \\
\text { preguntaría? o ¿qué le gustaría saber? }\end{array}$ \\
\hline
\end{tabular}

Para el grupo de educación para la salud se preparó una presentación en PowerPoint con fotografías en las que se daba al paciente y a su cuidador primario información acerca del cateterismo cardiaco, describiendo cada evento que viviría: la preparación previa al procedimiento (ayuno, colocación de vendajes y suero intravenoso), la descripción de la sala de hemodinamia en la que se realizaría la intervención, la preparación y administración de la anestesia, la realización del cateterismo, el paso a la sala de recuperación y a la habitación una vez finalizado el procedimiento. También se les proporcionó información respecto a los cuidados generales y los síntomas de alarma durante la recuperación dentro del hospital, al igual que una vez que se les diese de alta, destacando en todo momento la necesidad de seguir las indicaciones médicas y los riesgos que implicaba no hacerlo.

La información fue validada por personal experto médico y de enfermería; así como también por un grupo de adolescentes y sus cuidadores primarios que ya habían vivido esa experiencia, preguntándoles el tipo de información que les hubiera servido o qué les hubiera gustado saber. El personal de salud sugirió que las fotografías mostraran únicamente el campo de la incisión.

\section{Procedimiento}

Con previa autorización de las autoridades del hospital, se procedió a localizar a los participantes que cumplieran con los criterios de inclusión.

La asignación a los grupos fue por orden de aparición, respetando la elección del adolescente y de su cuidador principal en cuanto a recibir o no la educación para la salud; pasando la asignación al siguiente participante.

Hecho lo anterior, una psicóloga se presentaba a los adolescentes y a sus cuidadores primarios, a quienes invitaba a participar explicándoles el propósito del estudio y entregándoles a estos últimos una carta de consentimiento informado, así como otra de asentimiento informado a los adolescentes. En esos documentos se establecía el uso de la información obtenida, el carácter voluntario de su participación, así como que rehusar o retirar su participación no implicaba ninguna consecuencia respecto al tratamiento del adolescente ni al trato con el familiar. Se respondía cualquier duda y, en caso de estar de acuerdo, autorizaban su participación mediante su firma.

La psicóloga brindó la educación para la salud a los adolescentes y cuidadores primarios en 
conjunto y con apoyo de la presentación en PowerPoint, aclarando sus dudas en todo momento. Este proceso se llevó a cabo en la habitación asignada, sin interrupciones, con buena iluminación y comodidad para todos los participantes.

Los cuestionarios en donde se exploraba la utilidad y efecto de la educación para la salud se proporcionaron tanto a los adolescentes como a sus cuidadores primarios de ambos grupos momentos antes de su alta hospitalaria. Se revisaba que estuvieran completamente contestados y, en caso de duda, se aclaraba en ese momento la información proporcionada.

\section{Análisis de la información}

Las respuestas de los cuestionarios se transcribieron literalmente a un formato Excel, utilizándose la verificación de jueces ciegos independientes que verificaron aleatoriamente que la transcripción fuera fiel a las respuestas en el cuestionario en papel.

Se procedió a la categorización por contenidos centrales. En virtud de que la mayoría de las respuestas eran breves (de no más de dos o tres renglones), se emplearon términos clave como unidades de análisis. Se destacaban los términos que mejor representaban línea por línea el sentido de la respuesta. Luego se agruparon en categorías y se definieron según su importancia relativa en función de su frecuencia de aparición de términos indicativos mediante la técnica de análisis temático, tanto de la categoría principal, como de las categorías secundarias.

Para asegurar la familiaridad y un grado aceptable de validez social y ecológica en relación con los respondientes, la categorización estuvo a cargo de los propios autores del estudio, quienes estuvieron en contacto directo con los participantes (cf. Gale, Heath, Cameron, Rashid y Redwood, 2013).

\section{RESULTADOS}

Entre los adolescentes se observó una diferencia importante en cuanto a la apreciación de la utilidad de recibir información acerca del cateterismo cardiaco. En el GAH, 69.23\% consideró que le hubiera gustado tener la oportunidad de recibir información sobre el cateterismo cardiaco y su procedimiento, lo que le hubiera resultado útil, en contraste con la totalidad del GES, que la consideró útil. De manera similar, el GES obtuvo una calificación media de utilidad de 9 (D.E. $=1.55)$, en tanto que la del GAH fue de 6.38 (D.E. $=3.94$ ) (Tabla 2).

Tabla 2. Utilidad y calificación otorgada a la educación para la salud por los adolescentes y sus cuidadores primarios.

\begin{tabular}{|c|c|c|c|c|c|c|c|}
\hline & & \multicolumn{3}{|c|}{$\begin{array}{c}\text { Grupo con atención habitual } \\
\text { (Posible utilidad de la información) }\end{array}$} & \multicolumn{3}{|c|}{$\begin{array}{l}\text { Grupo con educación pra la salud } \\
\text { (Utilidad de la información recibida) }\end{array}$} \\
\hline & & Frecuencia & $\%$ & Calificación & Frecuencia & $\%$ & Calificación \\
\hline \multirow{3}{*}{ Adolescentes } & Sí es útil & 9 & 69.23 & Media: 6.38 & 19 & 100 & Media: 9 \\
\hline & No es útil & 4 & 30.77 & D.E.:3.94 & 0 & 0 & D.E.: 1.55 \\
\hline & Total & 13 & 100 & & 19 & 100 & \\
\hline \multirow{3}{*}{$\begin{array}{l}\text { Cuidadores } \\
\text { primarios }\end{array}$} & Sí es útil & 11 & 84.62 & Media: 6.08 & 19 & 100 & Media: 9.83 \\
\hline & No es útil & 2 & 15.38 & D.E.: 3.55 & 0 & & D.E.: 0.38 \\
\hline & Total & 13 & 100 & & 19 & 100 & \\
\hline
\end{tabular}

También se observaron diferencias en los cuidadores primarios: de $100 \%$ para los que recibieron educación para la salud, contra $84.62 \%$ que tuvieron atención habitual. En la calificación sobre su utilidad, se repitió la tendencia: $\mathrm{M}=9.83$, D.E. $=0.38$ vs. $\mathrm{M}=6.08$, D.E. $=3.55$, respectivamente. El grupo de cuidadores primarios percibió mayor utilidad en la posibilidad de haberla recibido de lo que lo hicieron los adolescentes en el GAH $(84.62 \%$ vs. 69.23\%) (Tabla 2).

Los adolescentes de ambos grupos calificaron como útil promover conductas orientadas a lograr un procedimiento exitoso (GES: $\mathrm{f}=29,61.7 \%$; GAH: $\mathrm{f}=8,34.78 \%$ ), así como conocer más sobre las etapas del cateterismo cardiaco y la autorregulación emocional (GES: $\mathrm{f}=18,38.29 \%$; GAH: 
$\mathrm{f}=11,47.82 \%)$ (estar tranquilo, seguro, menos triste) aunque en posiciones distintas, y en el GAH ( $\mathrm{f}=4,17.39 \%$ ) también se mencionó su inutilidad (derivación cognitiva de igualdad) (Figura 1).

Figura 1. Adolescentes con y sin educación para la salud.

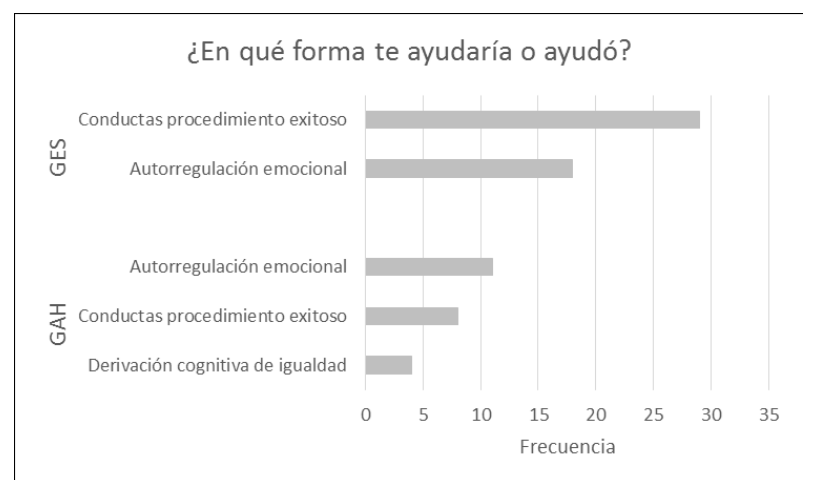

En este mismo sentido, los cuidadores primarios consideraron que la educación para la salud les ayudaría en primer lugar a mostrar conductas orientadas a un procedimiento exitoso (GES: $\mathrm{f}=23$, $51.11 \%$; GAH: $\mathrm{f}=15,65.21 \%$ ), como saber y poder decirle a sus hijos lo que es un cateterismo cardiaco, conocer los riesgos y saber cómo pueden ayudarlos durante el procedimiento médico. En segundo lugar, mencionaron su utilidad para la autorregulación emocional (GES: $\mathrm{f}=22,48.88 \%$; GAH: $\mathrm{f}=8,34.78 \%$ ), a fin de sentirse menos agobiados y más tranquilos, seguros y confiados (Figura 2).

Figura 2. Cuidadores primarios con y sin educación para la salud.

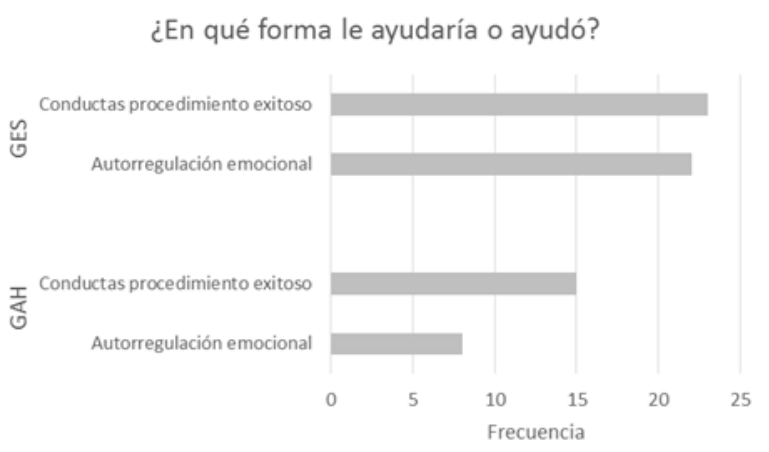

Ante la posibilidad de conversar con un adolescente ya cateterizado antes de someterse al procedimiento, los adolescentes de ambos grupos dijeron que, en primer lugar, les aconsejarían elegir cogniciones tales como no pensar en el dolor, que dicho procedimiento era fácil y rápido, que era para su bienestar y que recordaran la experiencia profesional de los médicos (GES: $\mathrm{f}=26,42.62 \%$; GAH: $\mathrm{f}=16,42.10 \%)$. También recomendarían conductas dirigidas a lograr un procedimiento exitoso (GES: $f=20,32.78 \%$; GAH: $f=6,15.78 \%$ ), como no moverse mucho, seguir las indicaciones del médico e informarse, entre otras, así como autorregularse emocionalmente (GES: $\mathrm{f}=14,22.95 \%$; GAH: $\mathrm{f}=15,39.47 \%$ ) y verbalizar su experiencia (GES: $f=1,1.63 \%$; GAH: $f=1,2.63 \%$ ).

Los cuidadores primarios de ambos grupos recomendarían a otros autorregularse emocionalmente (GES: $\mathrm{f}=19,44.18 \%$; GAH: $\mathrm{f}=12,30.77 \%$ ), estar tranquilos, tener fe, confianza, seguridad y paciencia. También promoverían las conductas dirigidas a lograr un procedimiento exitoso (GES: $f=12,27.90 \%$; GAH: $f=12,30.77 \%$ ) como el informarse, seguir las indicaciones del médico, motivar y platicar con su hijo, así como también promover la elección de cogniciones (GES: $\mathrm{f}=9$, 20.93\%; GAH: $f=12,30.77 \%$ ) (por ejemplo, "Es un procedimiento necesario, está con expertos y todo va a salir bien") y verbalizar la experiencia (GES: $\mathrm{f}=3,6.97 \%$; GAH: $\mathrm{f}=3,7.69 \%$ ).

Sobre qué le hubiera gustado preguntar al adolescente a otro que ya hubiera atravesado esa experiencia, se observó que ambos grupos preguntarían en primer lugar por las conductas destinadas a lograr un procedimiento exitoso (GES: $\mathrm{f}=29$, 72.50\%; GAH: $f=7,36.84 \%$ ) acerca de las etapas del cateterismo cardiaco, los cuidados que debían llevar a cabo y el nivel de dolor. También les gustaría saber lo que sintieron (autorregulación emocional) (GES: $\mathrm{f}=8,20 \%$; GAH: $\mathrm{f}=3,15.78 \%$ ) y que verbalizaran su experiencia (GES: $\mathrm{f}=1,2.5 \%$; GAH: $\mathrm{f}=2,10.52 \%$ ). Por otro lado, también hubo quienes dijeron no querer saber nada (derivación cognitiva de igualdad) (GES: $\mathrm{f}=2,5 \%$; GAH: $\mathrm{f}=4$, $21 \%)$. En el grupo con atención habitual también optaron por que se les dijera que todo iba a salir bien (elección de cogniciones) ( $\mathrm{f}=3,15.78 \%$ ).

Los cuidadores primarios de ambos grupos preguntarían en primer lugar a otros que ya pasaron por esa experiencia acerca de las conductas dirigidas a lograr un procedimiento exitoso (GES: $f=20,64.51 \%$; GAH: $f=17,65.38 \%$ ), los cuidados y riesgos del citado procedimiento, el nivel de dolor y la manera de ayudar a su hijo. También 
les gustaría saber cómo se sintieron (autorregulación emocional) (GES: $\mathrm{f}=5,16.12 \%$; GAH: $\mathrm{f}=1$, $3.84 \%$ ), que verbalizaran su experiencia (GES: $\mathrm{f}=5,16.12 \%$; GAH: $\mathrm{f}=1,3.84 \%$ ) y que se les dijera que todo saldría bien y que tuvieran confianza (elección de cogniciones) (GES: $\mathrm{f}=1,3.22 \%$; GAH: $\mathrm{f}=4,15.38 \%)$. En el grupo con atención habitual también dijeron no querer saber nada acerca de la intervención (derivación cognitiva de igual$\mathrm{dad})(\mathrm{f}=3,11.53 \%)$.

\section{DISCUSIÓN}

El presente trabajo trató de identificar la utilidad percibida de la educación para la salud por los adolescentes y por sus cuidadores primarios ante el cateterismo cardiaco. Ambos grupos consideraron que fue útil recibir información acerca del procedimiento médico al que iban a ser sometidos, aunque de manera más marcada entre los propios pacientes. La educación para la salud benefició las conductas orientadas a lograr un procedimiento exitoso y la autorregulación emocional, tanto en los adolescentes como en sus cuidadores primarios; sin embargo, en el grupo que recibió la atención habitual los adolescentes también mencionaron la inutilidad de este tipo de intervención.

El primer hallazgo difiere de lo reportado por Ghada, Zienab y Hossam (2016), en el que el protocolo educativo implementado logró una mejora significativa del conocimiento del cateterismo cardiaco para la colocación de stent en arteria coronaria; sin embargo, no la tuvo en las complicaciones circulatorias tras el cateterismo cardiaco.

Por otro lado, también se ha reportado que el proporcionar información altera aspectos emocionales tales como el nivel de miedo y la ansiedad, la disminución de molestias y el aumento de satisfacción en los pacientes cateterizados (Reed, Blake y Ohno-Machado, 2008; Samira et al., 2016), hallazgos que coinciden con lo reportado en el presente estudio.

Se ha observado asimismo que en los padres que reciben información respecto al tratamiento médico que se le hará a sus hijos no solamente se reducen los niveles de ansiedad (Watson y Visram, 2003), sino que también tienen un mejor ajuste a la situación, ya sea durante y después del proce- dimiento (Claar, Walker y Barnard, 2002). Estos resultados también se corroboran en la presente investigación; los cuidadores primarios con educación para la salud tenían la posibilidad de entender mejor lo que es un cateterismo cardiaco, logrando así un mejor control emocional y dirigiendo su atención a ayudar a sus hijos adolescentes, lo que implica un mayor involucramiento y sugiere un aumento de la capacidad para resolver problemas (conductas dirigidas a un procedimiento exitoso), que fue la principal utilidad reconocida por los cuidadores; lo que el grupo con atención habitual esperaba lograr al recibir este tipo de instrucción, adquiriendo así un mayor control emocional.

No obstante, adolescentes y cuidadores primarios de ambos grupos dieron respuestas acerca de lo contraproducente que resultaría recibir información, lo que se observó con mayor frecuencia en el grupo con atención habitual respecto a la posibilidad de haberla recibido. Si bien llama la atención la percepción negativa sobre la educación para la salud, principalmente porque afectaría su autorregulación emocional, no necesariamente implica evitar aquélla. Pese a ello, quienes eligen no recibir información sobre el padecimiento podrían tener problemas más importantes en lo concerniente a la adhesión terapéutica. En el estudio de Brito et al., (2016), hecho con pacientes de trasplante de riñón, aquellos sin adherencia a la medicación inmunosupresora empleaban más estrategias pasivas, como la evitación y pensamiento optimista, en comparación con los grupos con una adecuada adherencia, quienes utilizan más estrategias de afrontamiento centradas en el problema y la búsqueda de apoyo. Otro estudio hecho con pacientes en hemodiálisis mostró que quienes empleaban estrategias de afrontamiento centrado en el problema tenían mayores tasas de supervivencia y mejor salud física y mental que aquellos que utilizaban un estilo de afrontamiento centrado en la emoción (Niihata, Fukuma, Akizawa y Fukuhara, 2017). Es posible suponer que quienes estaban dispuestos a recibir información tendrían también estrategias de afrontamiento centradas en el problema, aunque el aspecto emocional también destacó como una cuestión importante en ambas condiciones. 
En este sentido, los adolescentes que recibieron la información mostraron tener un mayor control sobre la situación y más conductas orientadas al autocuidado durante su recuperación, entendiendo la necesidad de hacerlo así. A su vez, en el grupo sin educación para la salud se observaron más molestias y más quejas sobre el dolor y al momento de orinar. Lo anterior se atribuye a que los primeros sabían en qué consiste un cateterismo cardiaco, cómo es el proceso de anestesia, en qué condiciones iban a estar durante su recuperación $\mathrm{y}$, sobre todo, qué consecuencias podrían tener al no seguir las indicaciones médicas. Al parecer, saber cómo actuar y contar con la información sobre lo que podían hacer ante las situaciones de riesgo y cómo las podían evitar, permitió una mayor colaboración. Este hallazgo también se reportó en el estudio de Rivas, Maldonado, Caldera y Tepeyac (1999) sobre la importancia de la mayor colaboración en la recuperación de pacientes con cateterismo cardiaco informados.

Respecto a las recomendaciones que los adolescentes harían a otros adolescentes, se encontró que quienes recibieron la información se centraron más en la situación y en la necesidad de someterse al cateterismo cardiaco, generando con ello una elección de cogniciones y llevando a cabo conductas dirigidas a lograr un procedimiento exitoso, a diferencia del grupo con atención habitual, quienes empleaban más argumentos de elección de cogniciones y autorregulación emocional. En cuanto a los padres, se apreció que quienes recibieron la instrucción recomendarían sobre todo la autorregulación emocional, destacando la fe y la confianza, así como el uso de conductas dirigidas a lograr un procedimiento exitoso, acciones que en el grupo de atención habitual aparecen en segundo y tercer lugar.

Diferentes estudios señalan que los padres recurren a su espiritualidad para experimentar sentimientos de esperanza, fortaleza, confort y sentido de control sobre la situación (Cardella y Friedlander, 2004; Schneider y Mannell, 2006; Wilson y Miles, 2001). También se ha reportado que en tales padres aumenta su motivación por participar en el cuidado de sus hijos, al tiempo que evalúan las opciones de tratamiento al disponer de una mayor información relacionada a la condición médica de sus hijos, contribuyendo así al uso de estrategias efectivas para su cuidado (Coulson y Knibb, 2007; Kalichman et al., 2003).

Es de notarse que tanto adolescentes como cuidadores primarios en ambos grupos contarían y preguntarían sobre la experiencia de otros como último recurso; este hecho llama la atención, ya que se ha reportado que compartir sentimientos y experiencias al sufrir una enfermedad ofrece la oportunidad de recibir apoyo emocional y de intercambiar estrategias para resolver y sobrellevar las situaciones que se desencadenan ante aquella, lo que es característico de nuestra población (Vega y González, 2009).

Una de las limitaciones del presente estudio fue que no se contó en el grupo de educación para la salud con una evaluación previa acerca de la utilidad de esta intervención, para así saber si hubo cambios en esta variable una vez que sus integrantes recibieron la instrucción. Otra limitante fue que no se contó con una guía de observación estructurada que permitiera evaluar la utilidad de la educación para la salud en el seguimiento puntual de las instrucciones por parte de los adolescentes y cuidadores primarios y así poder realizar las comparaciones con grupos más grandes.

En futuros estudios se sugiere llevar a cabo un registro observacional e incluir indicadores fisiológicos del estrés tanto en los pacientes como en los cuidadores primarios; al igual que estudiar el impacto de la educación para la salud recibida en subsecuentes cateterismos, toda vez que a lo largo de su vida sufrirán más de una intervención de este tipo. También sería importante explorar si se modifican los estilos de afrontamiento en ambos tipos de participantes en aquellos casos en los que, dada la condición médica del adolescente, hayan experimentado más de un cateterismo cardiaco.

\section{REFERENCIAS}

Abbas, A.H. y Halima, Y.K. (2017). Effectiveness of educational video intervention on anxiety level of patients prior to diagnostic coronary catheterization in Al-Nasiriya's cardiac center. Journal of Nursing and Health Science, 6(4), 57-64. 
Aranha, P.R., Sams, L.M. y Saldanha, P. (2016). Impact of preoperative education program on parental anxiety: A pilot project. Archives of Medicine and Heatlh Sciences, 4(1), 30-34. doi: 10.4103/23214848.183374-.

Brito, D.C., Marsicano, E.O., Grincenkov, F.R., Colugnati, F.A., Lucchetti, G. y Sanders-Pinheiro, H. (2016). Stress, coping and adherence to immunosuppressive medications in kidney transplantation: a comparative study. Sao Paulo Medical Journal, 134(4): 292-299. doi: 10.1590/1516-3180.2015.01071008.

Buffum, M.D., Sasso, C., Sands, L.P., Lanier, E., Yellen, M. y Hayes, A. (2006). A music intervention to reduce anxiety before vascular angiography procedures. Journal of Vascular Nursing, 24(3), 68-73. doi: 10.1016/j.jvn.2006.04.001.

Cardella, L.A. y Friedlander, M.L. (2004). The relationship between religious coping and psychological distress in parents of children with cancer. Journal of Psychosocial Oncology, 22, 19-37. doi: 10.1300/J077v22n01_02.

Chair, S.Y., Chau, M.Y., Sit, J.W., Wong, E.M. y Chan, A.W. (2012). The psychological effects of a videotape educational intervention on cardiac catheterization patients. Contemporary Nurse, 40(2), 225-233. doi: 10.5172/conu.2012.40.2.225.

Claar, R.L., Walker, L.S. y Barnard, J.A. (2002). Children's knowledge, anticipatory anxiety, procedural distress, and recall of esophagogastroduodenoscopy. Journal of Pediatric Gastroenterology and Nutrition, 34(1), 68-72. doi: 10.1097/00005176200201000-00016.

Coulson, N.S. y Knibb, R.C. (2007). Coping with food allergy: Exploring the role of the online support group. Cyber Psychology \& Behavior, 10(1), 145-148. doi: 10.1089/cpb.2006.9978.

Ellerton, M.L. y Merriam, C. (1994). Preparing children and families psychologically for day surgery: an evaluation. Journal of Advanced Nursing, 19(6), 1057-1062. doi: 10.1111/j.1365-2648.1994.tb01188.x.

Elsobky, F.A., Mohamed, A.S. y Mohamend, S.A. (2018). The effect of pre-hospital discharge care program on mothers' knowledge and reported practice for children after congenital heart surgery. Journal of Nursing Education and Practice, 8(9), 122-130. doi:10.5430/jnep.v8n9p122.

Fernandes, S.C., Arriaga, P. y Esteves. F. (2014). Providing preoperative information for children undergoing surgery: a randomized study testing different types of educational material to reduce children's preoperative worries. Health Education Research, 29(6), 1058-1076. doi: 10.1093/her/cyu066.

Gale, K., Heath, G., Cameron, E., Rashid, S. y Redwood, S. (2013). Using the frame work method for the analysis of qualitative data in multidisciplinary health research. Bio Med Central Research Methodology, 13(117). Recuperado de http://www. ncbi.nlm.nih.gov/pmc/articles/PMC3848812/.

Ghada, T.M., Zienab, A.M. y Hossam, A.A. (2016). Impact of designed nursing educational protocol in health promotion for patients undergoing coronary artery stent outcome. Journal of Nursing and Health Science, 5(2), 54-63. doi: 10.9790/19590502065463 .

He, H.G., Zhu, L., Li, H.C., Wang, W., Vehviläinen, J.K. y Chan, S.W. (2014). A randomized controlled trial of the effectiveness of a therapeutic play intervention on outcomes of children undergoing in patient elective surgery: study protocol. Journal of Advanced Nursing, 70(2), 431-442. doi: 10.1111/jan.12234.

Jamshidi, N., Abbaszadeh, A. y Kaliani, M.N. (2010). The effects of video education on comfort and tolerability of patients undergoing coronary angiography. Iranian Journal of Nursing Research, 5(16), 38-44. URL: http://ijnr.ir/article-1-589-en.html.

Kalichman, S.C., Benotsch, E.G., Weinhardt, L., Austin, J., Luke, W. y Cherry, C. (2003). Health-related Internet use, coping, social support, and health indicators in people living with HIV/AIDS: Preliminary results from a community survey. Health Psychology, 22, 111-116. doi: 10.1037/0278-6133.22.1.111.

Keeling, A.W., Fisher, C.A., Haugh, K.H., Powers, E.R. y Turner, M.S. (2000). Reducing time in bed after percutaneous transluminal angioplasty (TIBS III). American Journal of Critical Care, 9(3), 185-187.

Kobayashi, D., Turner, D.R., Forbes, T.J. y Aggarwal, S. (2018). Parental anxiety among children undergoing cardiac catheterization. Cardiology in the Young, 28(2), 315-321. doi: 10.1017/S1047951117002074.

Lee, S. y Kim, S.S. (2010). The life of adolescent patients with complex congenital heart disease. Journal of Korean Academy of Nursing, 40(3), 411-422. doi: 10.4040/jkan.2010.40.3.411.

Mitchell, M. (2003). Patient anxiety and modern elective surgery: A literature review. Journal of Clinical Nursing, 12, 806-815.

Mu, P.F., Ma., F.C., Hwang, B. y Chao, Y.M. (2002). Families of children with cancer: the impact on anxiety experienced by fathers. Cancer Nursing, 25(1), 66-73.

Niihata, K., Fukuma, S., Akizawa, T. y Fukuhara, S. (2017). Association of coping strategies with mortality and health-related quality of life in hemodialysis patients: The Japan dialysis outcomes and practice patterns study, PLoSOne, 12(7), e0180498. doi: 10.1371/journal.pone.0180498. eCollection 2017.

Peterson, J.C., Allegrante, J.P., Pirraglia, P.A., Robbins, L., Lane, P., Boschert, K.A. y Charlson, M.E. (2010). Living with heart disease after angioplasty: A qualitative study of patients who have been successful or unsuccessful in multiple behavior change. Heart \& Lung, 39(2), 105-115. doi: 10.1016/j.hrtlng.2009.06.017.

Reed, F.S., Blake, G. y Ohno-Machado, L. (2008). Vascular closure devices and the risk of vascular complications after percutaneous coronary intervention in patients receiving glycoprotein IIb-IIIa inhibitors. American Journal of Cardiolology, 88, 493-496. 
Rezaei-Adaryani, M., Ahmadi, F., Mohamadi, E. y Asghari-Jafarabadi, M. (2009). The effect of three positioning methods on patient outcomes after cardiac catheterization. Journal of Advanced Nursing, 65(2), 417-424. doi: 10.1111/j.13652648.2008.04889.x.

Rivas E., J., Maldonado N., E., Caldera L., D. y Tepeyac L., A. (1999). Disminución del riesgo de lesión por sangrado en el sitio de punción al adulto sometido a cateterismo cardiaco mediante: satisfacción del déficit de conocimiento e inmovilidad. Revista Mexicana de Enfermería Cardiológica, 7(1-4), 12-18.

Rodríguez G., M., Arredondo H., E. y Herrera C., R. (2012). Efectividad de un programa educativo en enfermería en el autocuidado de los pacientes con insuficiencia cardíaca: ensayo clínico controlado. Revista Latino Americana Enfermagem, 20(2), 296-306.

Samira, E.A., Nahid, F.G. y Samah, E.M. (2016). Effect of early nursing preparation on anxiety among patients undergoing cardiac catheterization. American Journal of Nursing Science, 5(5), 222-231. doi: 10.11648/j.ajns.20160505.17.

Schneider, M.A. y Mannell, R.C. (2006). Beacon in the storm: An exploration of the spirituality and faith of parents whose children have cancer. Issues in Comprehensive Pediatric Nursing, 29, 3-24. doi: 10.1080/01460860500523731.

Utens, E., Versluis-Den, H.J., Verhulst, F.C., Witsenburg, M., Borges, A.J. yHess, J. (2000). Psychological distress and styles of coping in parents of children awaiting elective cardiac surgery. Cardiology in the Young, 10(3), 239-244.

Vega, A.O. y González, E.D. (2009). Apoyo social: elemento clave en el afrontamiento de la enfermedad crónica. Enfermería Global, 16, 1-11.

Vincent, C., Chiappetta, M., Beach, A., Kiolbasa, C., Latta, K., Maloney, R. y Van Roeyen, L.S. (2012). Parents' management of children's pain at home after surgery. Journal for Specialists in Pediatric Nursing, 17(2), 108-120. doi: 10.1111/j.17446155.2012.00326.x.

Watson, A.T. y Visram, A. (2003). Children's preoperative anxiety and postoperative behaviour. Paediatric Anaesthesia, 13(3), 188-204. doi: 10.1046/j.1460-9592.2003.00848.x.

Wilson, S.M. y Miles, M.S. (2001). Spirituality in African-American mothers coping with a seriously ill infant. Journal for Specialists in Pediatric Nursing, 6, 116-122.

Woods, S.L., Froelicher, E.S.S., Motzer, S.U. y Bridges, E.J. (2010). Cardiac Nursing (6th ed.) Philadelphia, PA: Lippincott. 